\title{
AN APPROACH TO SOLUTION OF THE SCHRÖDINGER EQUATION USING FOURIER-TYPE FUNCTIONALS
}

\author{
Seung Jun Chang, Jae Gil Choi, and Hyun Soo Chung
}

\begin{abstract}
In this paper, we consider the Fourier-type functionals on Wiener space. We then establish the analytic Feynman integrals involving the $\diamond$-convolutions. Further, we give an approach to solution of the Schrödinger equation via Fourier-type functionals. Finally, we use this approach to obtain solutions of the Schrödinger equations for harmonic oscillator and double-well potential. The Schrödinger equations for harmonic oscillator and double-well potential are meaningful subjects in quantum mechanics.
\end{abstract}

\section{Introduction}

Let $C_{0}[0, T]$ denote the one-parameter Wiener space, that is, the space of continuous real-valued functions $x$ on $[0, T]$ with $x(0)=0$. In 1948, Feynman assumed the existence of an integral over a space of paths and used this integral in a formal way in his approach to quantum mechanics [9]. A number of mathematicians have attempted to give rigorously meaningful definitions of the Feynman integral with appropriate existence theorems and have expressed solutions of the Schrödinger equation in terms of their integrals. One of these approaches is based on the similarity between the Wiener and the Feynman integrals, where procedures are developed to obtain the Feynman integrals from the Wiener integrals by an analytic extension from the real axis to the imaginary axis.

Consider a differential equation

$$
\frac{\partial}{\partial t} \psi(u, t)=\frac{1}{2 \lambda} \Delta \psi(u, t)-V(u) \psi(u, t)
$$

with the initial condition $\psi(u, 0)=\varphi(u)$, where $\Delta$ is the Laplacian and $V$ is an appropriate potential function. For $\lambda>0$, this is the diffusion equation with

Received December 6, 2011.

2010 Mathematics Subject Classification. Primary 60J65, 81Q05, 28C20, 46C07.

Key words and phrases. Schrödinger equation, harmonic oscillator, double-well potential, Fourier-type functional, Feynman-Kac formula, Fourier transform.

This research was supported by Basic Science Research Program through the National Research Foundation of Korea(NRF) funded by the Ministry of Education, Science and Technology(2012R1A1A1004774; 2011-0014552). 
the potential $V(u)$. From the Feynman-Kac formula we know that the solution of equation (1.1) can be written as a Wiener integral

$$
\int_{C_{0}[0, T]} \varphi\left(\lambda^{-1 / 2} x(T)\right) \exp \left\{-\int_{0}^{T} V\left(\lambda^{-1 / 2} x(s)\right) d s\right\} d m(x) .
$$

It is a well-known fact that when the time is replaced with the imaginary time, this diffusion equation becomes the Schrödinger equation

$$
i \frac{\partial}{\partial t} \psi(u, t)=-\frac{1}{2} \Delta \psi(u, t)+V(u) \psi(u, t)
$$

with the initial condition $\psi(u, 0)=\varphi(u)$. Hence the solution of the Schrödinger equation (1.2) is obtained via the analytic Feynman integral.

For a more detailed study, see $[9,10,12,13,14,15,19,21]$.

In [7], the authors introduced the Fourier-type functionals via the Fourier transform and studied some properties of the Fourier-type functionals. The Fourier-type functionals are useful in applications of the analytic Feynman integral to quantum theory. In this paper, we obtain a solution of the Schrödinger equation (1.2) which is the limit of the analytic Feynman integrals for the Fourier-type functionals. We then use our results to obtain solutions of the Schrödinger equations for harmonic oscillator and double-well potential. Finally, we give some applications as remarks.

\section{Definitions and preliminaries}

In this section, we list some definitions and results from $[7,8,20]$.

Throughout this paper, we will assume that each functional $F: C_{0}[0, T] \rightarrow \mathbb{C}$ we consider is scale-invariant measurable and that $\int_{C_{0}[0, T]}|F(\rho x)| d m(x)<\infty$ for each $\rho>0$ (see [11]).

First, we state the definition of the analytic Feynman integral.

Definition 2.1. Let $\mathbb{C}$ denote the complex numbers, let $\mathbb{C}_{+}=\{\lambda \in \mathbb{C}$ : $\operatorname{Re}(\lambda)>0\}$ and let $\tilde{\mathbb{C}}_{+}=\{\lambda \in \mathbb{C}: \lambda \neq 0$ and $\operatorname{Re}(\lambda) \geq 0\}$. Let $F: C_{0}[0, T] \rightarrow$ $\mathbb{C}$ be a measurable functional such that for each $\lambda>0$, the Wiener integral

$$
J(\lambda)=\int_{C_{0}[0, T]} F\left(\lambda^{-1 / 2} x\right) d m(x)
$$

exists. If there exists a function $J^{*}(\lambda)$ analytic in $\mathbb{C}_{+}$such that $J^{*}(\lambda)=J(\lambda)$ for all $\lambda>0$, then $J^{*}(\lambda)$ is defined to be the analytic Wiener integral of $F$ over $C_{0}[0, T]$ with parameter $\lambda$, and for $\lambda \in \mathbb{C}_{+}$, we write

$$
J^{*}(\lambda)=\int_{C_{0}[0, T]}^{\mathrm{an}_{\lambda}} F(x) d m(x) .
$$

Let $q \neq 0$ be a real number and let $F$ be a functional such that $J^{*}(\lambda)$ exists for all $\lambda \in \mathbb{C}_{+}$. If the following limit exists, we call it the analytic Feynman 
integral of $F$ with parameter $q$ and write

$$
\int_{C_{0}[0, T]}^{\operatorname{anf}_{q}} F(x) d m(x)=\lim _{\lambda \rightarrow-i q} \int_{C_{0}[0, T]}^{\mathrm{an}_{\lambda}} F(x) d m(x),
$$

where $\lambda \rightarrow-i q$ through values in $\mathbb{C}_{+}$.

For $v \in L_{2}[0, T]$ and $x \in C_{0}[0, T]$, let $\langle v, x\rangle$ denote the Paley-WienerZygmund (PWZ) stochastic integral. One can show that for each $v \in L_{2}[0, T]$, $\langle v, x\rangle$ exists for a.e. $x \in C_{0}[0, T]$ and if $v \in L_{2}[0, T]$ is a function of bounded variation on $[0, T]$, then $\langle v, x\rangle$ equals the Riemann-Stieltjes integral $\int_{0}^{T} v(t) d x(t)$ for s-a.e. $x \in C_{0}[0, T]$. Also, $\langle v, x\rangle$ has the expected linearity property. Furthermore, $\langle v, x\rangle$ is a Gaussian process with mean 0 and variance $\|v\|_{2}^{2}$. For a more detailed study of the PWZ stochastic integral, see $[5,6,7,8]$.

The following theorem is a well-known integration formula which is to be used several times throughout the present paper.

Theorem 2.2. Let $\left\{\alpha_{1}, \ldots, \alpha_{n}\right\}$ be an orthonormal set of functions from $L[0, T]$. Let $f: \mathbb{R}^{n} \rightarrow \mathbb{C}$ be Borel measurable, and let

$$
F(x)=f\left(\left\langle\alpha_{1}, x\right\rangle, \ldots,\left\langle\alpha_{n}, x\right\rangle\right) \equiv f(\langle\vec{\alpha}, x\rangle) .
$$

Then

$$
\begin{aligned}
\int_{C_{0}[0, T]} F(x) d m(x) & =\int_{C_{0}[0, T]} f(\langle\vec{\alpha}, x\rangle) d m(x) \\
& =\left(\frac{1}{2 \pi}\right)^{\frac{n}{2}} \int_{\mathbb{R}^{n}} f(\vec{v}) \exp \left\{-\frac{|\vec{v}|^{2}}{2}\right\} d \vec{v},
\end{aligned}
$$

where $|\vec{v}|=\sqrt{v_{1}^{2}+\cdots+v_{n}^{2}}$ in the sense that if either side of (2.1) exists, then both sides exist and the equality holds.

Now we state some well-known properties of the Fourier transform as a lemma (see [20]).

Lemma 2.3. (1) Let $f \in C^{\infty}\left(\mathbb{R}^{n}\right)$ be such that all Laplacians $\Delta^{k} f, k=$ $0,1, \ldots$, belong to $L_{2}\left(\mathbb{R}^{n}\right)$. Then

$$
\widehat{\Delta^{k} f}(\vec{\xi})=(-1)^{k}|\vec{\xi}|^{2 k} \hat{f}(\vec{\xi})
$$

where $\widehat{f}$ is the Fourier transform of $f$,

$$
\widehat{f}(\vec{\xi})=\left(\frac{1}{2 \pi}\right)^{\frac{n}{2}} \int_{\mathbb{R}^{n}} f(\vec{u}) \exp \{i \vec{u} \cdot \vec{\xi}\} d \vec{u}, \quad \vec{u}, \vec{\xi} \in \mathbb{R}^{n},
$$

and $\vec{u} \cdot \vec{\xi}=u_{1} \xi_{1}+\cdots+u_{n} \xi_{n}$.

(2) The following statement is called the Paley-Wiener-type theorem for the Fourier transform on $\mathbb{R}^{n}$. A function $f$, square integrable on $\mathbb{R}^{n}$, is the restriction on $\mathbb{R}^{n}$ of an entire function of exponential type if and only if $\Delta^{k} f$ belong to $L_{2}\left(\mathbb{R}^{n}\right)$ for all positive integers $k$. 
Let $\mathcal{S}\left(\mathbb{R}^{n}\right)$ be the Schwartz space of infinitely differentiable function $f(\vec{u})$ decaying at infinity together with all its derivatives faster than any polynomial of $|\vec{u}|^{-1}$. Note that the Fourier transform is an isomorphism on the Schwartz space $\mathcal{S}\left(\mathbb{R}^{n}\right)$. Also, $\Delta^{k} f$ and $\widehat{\Delta^{k} f}$ are elements on $\mathcal{S}\left(\mathbb{R}^{n}\right)$ for all $k=1,2, \ldots$

Now we introduce the Fourier-type functionals defined on $C_{0}[0, T]$ (see [7]).

Definition 2.4. Let $\left\{\alpha_{1}, \ldots, \alpha_{n}\right\}$ be an orthonormal set of functions from $L_{2}[0, T]$. For $f \in \mathcal{S}\left(\mathbb{R}^{n}\right)$, let

$$
\Delta^{k} F(x)=\left(\Delta^{k} f\right)(\langle\vec{\alpha}, x\rangle), \quad k=0,1, \ldots
$$

$$
\widehat{\Delta^{k} F}(x)=\widehat{\Delta^{k}} f(\langle\vec{\alpha}, x\rangle), \quad k=0,1, \ldots
$$

The functionals in (2.4) and (2.5) are called the Fourier-type functionals defined on Wiener space $C_{0}[0, T]$.

We obtain a useful formula for the Fourier-type functionals which will be used in the last section. Using equation (2.2), the Fourier-type functional $\widehat{\Delta^{k} F}$ given in equation (2.5) can be expressed by

$$
\widehat{\Delta^{k} F}(x)=(-1)^{k}|\langle\vec{\alpha}, x\rangle|^{2 k} \widehat{F}(x)
$$

for all $k=0,1, \ldots$

\section{Feynman integral for the Fourier-type functionals}

In this section, we evaluate the analytic Fenyman integrals for the Fouriertype functionals.

Theorem 3.1. Let $\widehat{\Delta^{k} F}$ be defined as in equation (2.5). Then for all $q \in$ $\mathbb{R}-\{0\}$, the analytic Feynman integral of $\widehat{\Delta^{k} F}$ exists and is given by the formula

$$
\int_{C_{0}[0, T]}^{\operatorname{anf}_{q}} \widehat{\Delta^{k} F}(x) d m(x)=\left(\frac{1}{2 \pi}\right)^{\frac{n}{2}} \int_{\mathbb{R}^{n}}\left(\Delta^{k} f\right)(\vec{v}) \exp \left\{-\frac{i|\vec{v}|^{2}}{2 q}\right\} d \vec{v}
$$

for all $k=0,1, \ldots$.

Proof. By using equations (2.1) and (2.3), we have that for all $\lambda>0$,

$$
\begin{aligned}
& \int_{C_{0}[0, T]} \widehat{\Delta^{k} F}\left(\lambda^{-1 / 2} x\right) d m(x) \\
= & \left(\frac{\lambda}{2 \pi}\right)^{\frac{n}{2}}\left(\frac{1}{2 \pi}\right)^{\frac{n}{2}} \int_{\mathbb{R}^{n}} \int_{\mathbb{R}^{n}}\left(\Delta^{k} f\right)(\vec{v}) \exp \{i \vec{v} \cdot \vec{\xi}\} d \vec{v} \exp \left\{-\frac{\lambda|\vec{\xi}|^{2}}{2}\right\} d \vec{\xi} \\
= & \left(\frac{1}{2 \pi}\right)^{\frac{n}{2}} \int_{\mathbb{R}^{n}}\left(\Delta^{k} f\right)(\vec{v}) \exp \left\{-\frac{|\vec{v}|^{2}}{2 \lambda}\right\} d \vec{v} .
\end{aligned}
$$


By using Morera's theorem, the last expression above is an analytic function of $\lambda \in \mathbb{C}_{+}$and is a continuous function of $\lambda \in \widetilde{\mathbb{C}}_{+}$, which, by letting $\lambda \rightarrow i q$, yields equation (3.1). Furthermore, we obtain that

$$
\left|\int_{C_{0}[0, T]}^{\operatorname{anf}_{q}} \widehat{\Delta^{k} F}(x) d m(x)\right| \leq \int_{\mathbb{R}^{n}}\left|\left(\Delta^{k} f\right)(\vec{v})\right| d \vec{v}<\infty
$$

for all $q \in \mathbb{R}-\{0\}$. Hence we complete the proof of Theorem 3.1 as desired.

Recall that the convolution of the Fourier transform has many useful basic properties. Many mathematicians have used these properties to solve appropriate differential equations in their fields. In Theorem 3.1, we considered the Fourier-type functional via the Fourier transform. So we need a new concept that resembles the convolution of the Fourier transform.

Next we give the definition of $\diamond$-convolution of functionals on $C_{0}[0, T]$.

Definition 3.2. Let $F$ be defined as in Definition 2.4 and let $G$ be the Fouriertype functional on $C_{0}[0, T]$ of the form

$$
G(x)=g(\langle\vec{\alpha}, x\rangle) .
$$

We define their $\diamond$-convolution by

$$
(F \diamond G)(x)=(f * g)(\langle\vec{\alpha}, x\rangle) .
$$

The $\diamond$-convolution preserves a useful property of convolution for the Fourier transform as follows. Let $F$ and $G$ be the Fourier-type functionals on $C_{0}[0, T]$. Then the $\diamond$-convolution $(F \diamond G)$ of $F$ and $G$ always exists since $f$ and $g$ are elements of $\mathcal{S}\left(\mathbb{R}^{n}\right)$ and

$$
(\widehat{F \diamond G})(x)=(2 \pi)^{\frac{n}{2}} \widehat{F}(x) \widehat{G}(x)=(\widehat{G \diamond F})(x) .
$$

Equation (3.2) plays a key role in this paper.

In our next theorem, we establish the analytic Feynman integral for the $\diamond$-convolution of $F$ and $G$.

Theorem 3.3. Let $F$ and $G$ be defined as in Definition 3.2. Then for all $q \in \mathbb{R}-\{0\}$, the analytic Feynman integral of $(F \diamond G)$ exists and

$$
\int_{C_{0}[0, T]}^{\operatorname{anf}_{q}}(\widehat{F \diamond G})(x) d m(x)=(2 \pi)^{\frac{n}{2}} \int_{C_{0}[0, T]}^{\operatorname{anf}_{q}} \widehat{F}(x) \widehat{G}(x) d m(x) .
$$

Also, both sides of the above equation are given by the formula

$$
\left(\frac{1}{2 \pi}\right)^{\frac{n}{2}} \int_{\mathbb{R}^{n}}(f * g)(\vec{v}) \exp \left\{-\frac{i|\vec{v}|^{2}}{2 q}\right\} d \vec{v} .
$$

Proof. From Theorem 3.1, the left-hand side of equation (3.3) exists since $\widehat{(f * g)} \in \mathcal{S}\left(\mathbb{R}^{n}\right)$. Also, using equation (3.2), we establish equation (3.3) as desired. Equation (3.4) follows by letting $k=0$ and replacing $\widehat{\Delta F}$ with $(\widehat{F \diamond G})$ in equation (3.1). Hence we complete the proof of Theorem 3.3 as desired. 


\section{A solution of the Schrödinger equation}

In this section, we will show that the solution of the Schrödinger equation (1.2) is the limit of the analytic Feynman integrals for the Fourier-type functionals under appropriate conditions.

From the definitions of the Fourier-type functionals and the analytic Feynman integral, we obtain some important observations. Let

$$
F(x) \equiv \varphi(x(T)) \exp \left\{-\int_{0}^{T} V(x(s)) d s\right\},
$$

where $\varphi$ and $V$ are as in equation (1.1). Then the solution of the diffusion equation (1.1) is the Wiener integral

$$
\int_{C_{0}[0, T]} F\left(\lambda^{-\frac{1}{2}} x\right) d m(x)
$$

and the solution of the Schrödinger equation (1.2) is the analytic Feynman integral

$$
\int_{C_{0}[0, T]}^{\operatorname{anf}_{1}} F(x) d m(x)
$$

of $F$. Using these observations, we obtain a solution of the Schrödinger equation (1.2) which is the limit of the analytic Feynman integrals for the Fourier-type functionals. Before we do this, we need the following assumptions.

Assume that

$$
\exp \left\{-\int_{0}^{T} V(x(s)) d s\right\}=\lim _{m \rightarrow \infty} \widehat{V_{m}}(x)
$$

for a.e. $x \in C_{0}[0, T]$, where

$$
\widehat{V_{m}}(x)=\widehat{f_{m}}(\langle\alpha, x\rangle)
$$

and $f_{m}$ is an element of $\mathcal{S}\left(\mathbb{R}^{m}\right)$ for each $m=1,2, \ldots$ Then $\widehat{V_{m}}$ is a Fourier-type functional and $\widehat{V_{m}}$ is bounded on $C_{0}[0, T]$ for each $m=1,2, \ldots$. Let

$$
F_{m}(x) \equiv \varphi(x(T)) \widehat{V_{m}}(x) .
$$

Then we have

$$
\lim _{m \rightarrow \infty} F_{m}(x)=F(x)
$$

for a.e. $x \in C_{0}[0, T]$.

In our next lemma, we give a solution of the diffusion equation (1.1) which is the limit of the Wiener integrals for the Fourier-type functionals.

Lemma 4.1. Let $\varphi$ and $\widehat{V_{m}}$ be given by equations (1.1) and (4.3), respectively. Assume $\varphi$ is a bounded function. Then

$$
\lim _{m \rightarrow \infty} \int_{C_{0}[0, T]} \varphi\left(\lambda^{-\frac{1}{2}} x(T)\right) \widehat{V_{m}}\left(\lambda^{-\frac{1}{2}} x\right) d m(x)
$$

is a solution of the diffusion equation (1.1). 
Proof. By our assumption on $\varphi$ and the fact that $\left|\widehat{V_{m}}(x)\right| \leq M, M>0$ for all $m=1,2, \ldots$, we see that

$$
\varphi\left(\lambda^{-\frac{1}{2}} x(T)\right) \widehat{V_{m}}\left(\lambda^{-\frac{1}{2}} x\right)
$$

is bounded on $C_{0}[0, T]$. Hence using the Bounded Convergence Theorem and equations (4.2) and (4.4), we complete the proof of Lemma 4.1 as desired.

The following theorem is the first main result of this paper. The solution of the Schrödinger equation (1.2) is the limit of the analytic Feynman integrals for the Fourier-type functionals

Theorem 4.2. Let $\varphi$ and $\widehat{V_{m}}$ be as in Lemma 4.1. Then

$$
\lim _{m \rightarrow \infty} \int_{C_{0}[0, T]}^{\operatorname{anf}_{1}} \varphi(x(T)) \widehat{V_{m}}(x) d m(x)
$$

is a solution of the Schrödinger equation (1.2).

Proof. From Lemma 4.1, for $\lambda>0$,

$$
\int_{C_{0}[0, T]} \varphi\left(\lambda^{-\frac{1}{2}} x(T)\right) \widehat{V_{m}}\left(\lambda^{-\frac{1}{2}} x\right) d m(x)
$$

always exists. By using Morera's theorem, the last expression is an analytic function of $\lambda \in \mathbb{C}_{+}$and is a continuous function of $\lambda \in \widetilde{\mathbb{C}}_{+}$and so the analytic Feynman integral

$$
\int_{C_{0}[0, T]}^{\operatorname{anf}_{1}} \varphi(x(T)) \widehat{V_{m}}(x) d m(x)
$$

exists for all $m=1,2, \ldots$. Furthermore, by using the Bounded Convergence Theorem, we have

$$
\lim _{m \rightarrow \infty} \int_{C_{0}[0, T]}^{\operatorname{anf}_{1}} \varphi(x(T)) \widehat{V_{m}}(x) d m(x)=\int_{C_{0}[0, T]}^{\mathrm{anf}_{1}} F(x) d m(x),
$$

where $F$ is given by equation (4.1). Hence we have the desired result.

The following theorem is the second main result of this paper. We obtain an expression for the solution of Schrödinger equation (1.2) via the $\diamond$-convolution.

Theorem 4.3. Let $\varphi$ and $\widehat{V_{m}}$ be as in Theorem 4.2. Assume that $\varphi \in \mathcal{S}\left(\mathbb{R}^{\nu}\right)$ for all $\nu=1,2, \ldots$ and $\widehat{\Phi}$ is a Fourier-type functional so that $\widehat{\Phi}(x)=\varphi(x(T))$. Then

$$
\lim _{m \rightarrow \infty}(2 \pi)^{-\frac{m}{2}} \int_{C_{0}[0, T]}^{a^{a n f_{1}}}\left(\widehat{\Phi \diamond V_{m}}\right)(x) d m(x)
$$

is also a solution of the Schrödinger equation (1.2). 
Proof. Our assumptions and equation (3.3) tell us that, for each $m=1,2, \ldots$,

$$
\int_{C_{0}[0, T]}^{\text {anf }_{1}} \widehat{\Phi}(x) \widehat{V_{m}}(x) d m(x)=(2 \pi)^{-\frac{m}{2}} \int_{C_{0}[0, T]}^{\operatorname{anf}_{1}}\left(\widehat{\Phi \diamond V_{m}}\right)(x) d m(x) .
$$

Using Theorem 4.2, the limit of the left-hand side of equation (4.6) exists and hence we complete the proof of Theorem 4.3 as desired.

The following remark immediately follows from equation (4.6).

Remark 4.4. Let $\varphi, V_{m}, \Phi$ and $F$ be as in Theorem 4.3. Then

$$
\lim _{m \rightarrow \infty} \int_{C_{0}[0, T]}^{\operatorname{anf}_{1}} \widehat{\Phi}(x) \widehat{V_{m}}(x) d m(x)
$$

is also a solution of the Schrödinger equation (1.2).

\section{Applications}

In this section, we give some applications of our results from Section 4 that are meaningful subjects in quantum mechanics.

When $V(u)=a^{2} u^{2}, a \in \mathbb{R}-\{0\}$ in equation (1.1), this equation is called the diffusion equation for harmonic oscillator

$$
\frac{\partial}{\partial t} \psi(u, t)=\frac{1}{2 \lambda} \Delta \psi(u, t)-a^{2} u^{2} \psi(u, t)
$$

with the initial condition $\psi(u, 0)=\varphi(u)$ and hence the solution of the diffusion equation for harmonic oscillator is given by

$$
\int_{C_{0}[0, T]} \varphi\left(\lambda^{-1 / 2} x(T)\right) \exp \left\{-\frac{a^{2}}{\lambda} \int_{0}^{T} x^{2}(s) d s\right\} d m(x) .
$$

Also, when the time is replaced with the imaginary time, this diffusion equation for the harmonic oscillator (5.1) becomes the Schrödinger equation for the harmonic oscillator

$$
i \frac{\partial}{\partial t} \psi(u, t)=-\frac{1}{2} \Delta \psi(u, t)+a^{2} u^{2} \psi(u, t)
$$

with the initial condition $\psi(u, 0)=\varphi(u)$.

Throughout this section, we will show that the solution of the Schrödinger equation for harmonic oscillator (5.2) is the limit of the analytic Feynman integrals for the Fourier-type functionals. In order to do this, we will state some previous results from $[1,2,3,4]$ as follows.

Let

$$
C_{0}^{\prime}[0, T]=\left\{w \in C_{0}[0, T]: w(t)=\int_{0}^{t} w(s) d s, w \in L_{2}[0, T]\right\} .
$$

Then it is a separable infinite dimensional Hilbert space with inner product

$$
\left(w_{1}, w_{2}\right)_{C_{0}^{\prime}}=\int_{0}^{T} w_{1}^{\prime}(t) w_{2}^{\prime}(t) d t .
$$


As is known, $\left(C_{0}^{\prime}[0, T], C_{0}[0, T], m\right)$ is an example of an abstract Wiener space [16].

Let $S: C_{0}^{\prime}[0, T] \rightarrow C_{0}^{\prime}[0, T]$ be the linear operator defined by

$$
S w(t)=\int_{0}^{t} w(s) d s
$$

Then we see that the adjoint operator $S^{*}$ of $S$ is given by

$$
S^{*} w(t)=w(T) t-\int_{0}^{t} w(s) d s=\int_{0}^{t}[w(T)-w(s)] d s
$$

and the linear operator $A=S^{*} S$ is given by

$$
A w(t)=\int_{0}^{T} \min \{s, t\} w(s) d s .
$$

Furthermore, we see that $A$ is a self-adjoint operator on $C_{0}^{\prime}[0, T]$ and that

$$
\left(w_{1}, A w_{2}\right)_{C_{0}^{\prime}}=\left(S w_{1}, S w_{2}\right)_{C_{0}^{\prime}}=\int_{0}^{T} w_{1}(s) w_{2}(s) d s
$$

for all $w_{1}, w_{2} \in C_{a, b}^{\prime}[0, T]$. Hence $A$ is a positive definite operator, i.e.,

$$
(w, A w)_{C_{0}^{\prime}} \geq 0 \text { for all } w \in C_{a, b}^{\prime}[0, T] .
$$

One can show that the orthonormal eigenfunctions $\left\{e_{m}\right\}$ of $A$ are given by

$$
e_{m}(t)=\frac{\sqrt{2 T}}{\left(m-\frac{1}{2}\right) \pi} \sin \left(\frac{\left(m-\frac{1}{2}\right) \pi}{T} t\right) \equiv \int_{0}^{t} \alpha_{m}(s) d s
$$

with corresponding eigenvalues $\left\{\beta_{m}\right\}$ given by

$$
\beta_{m}=\left(\frac{T}{\left(m-\frac{1}{2}\right) \pi}\right)^{2} .
$$

Furthermore, it can be shown that $\left\{e_{m}\right\}$ is a basis of $C_{0}^{\prime}[0, T]$ and so $\left\{\alpha_{m}\right\}$ is a basis of $L_{2}[0, T]$, and that $A$ is a trace class operator and so $S$ is a HilbertSchmidt operator on $C_{0}^{\prime}[0, T]$. In fact, the trace of $A$ is given by $\operatorname{Tr} A=\frac{1}{2} T^{2}=$ $\int_{0}^{T} t d t$. In this case,

$$
\int_{0}^{T} x^{2}(s) d s=\lim _{m \rightarrow \infty} \sum_{j=1}^{m} \beta_{j}\left\langle\alpha_{j}, x\right\rangle^{2}
$$

for a.e. $x \in C_{0}[0, T]$.

The following lemma follows from equation (5.5).

Lemma 5.1. For each $n=1, \ldots$, let $\beta_{n}$ be as in equation (5.4). Then we obtain

$$
\lim _{m \rightarrow \infty} \exp \left\{-a^{2} \sum_{j=1}^{m} \beta_{j}\left\langle\alpha_{j}, x\right\rangle^{2}\right\}=\exp \left\{-a^{2} \int_{0}^{T} x^{2}(s) d s\right\}
$$

for a.e. $x \in C_{0}[0, T]$. 
We note that there is a function $f_{m}$ in $\mathcal{S}\left(\mathbb{R}^{m}\right)$ so that

$$
\widehat{f_{m}}(\vec{\xi})=\exp \left\{-a^{2} \sum_{j=1}^{m} \beta_{j} \xi_{j}^{2}\right\} \text {. }
$$

In fact, $f_{m}$ is given by the inverse Fourier transform of

$$
\exp \left\{-a^{2} \sum_{j=1}^{m} \beta_{j} \xi_{j}^{2}\right\} \text {. }
$$

Now, let $V_{m}(x)=f_{m}(\langle\vec{\alpha}, x\rangle)$. Then $V_{m}$ is a Fourier-type functional and so $\widehat{V_{m}}$ is also a Fourier-type functional. Furthermore, we obtain

$$
\widehat{V_{m}}(x)=\exp \left\{-a^{2} \sum_{j=1}^{m} \beta_{j}\left\langle\alpha_{j}, x\right\rangle^{2}\right\}
$$

and so, by using Lemma 5.1,

$$
\lim _{m \rightarrow \infty} \widehat{V_{m}}(x)=\exp \left\{-a^{2} \int_{0}^{T} x^{2}(s) d s\right\}
$$

for a.e. $x \in C_{0}[0, T]$. Also, for all $m=1,2, \ldots,\left|\widehat{V_{m}}(x)\right| \leq 1$ and

$$
\lim _{m \rightarrow \infty} \varphi(x(T)) \widehat{V_{m}}(x)=F(x)
$$

for a.e. $x \in C_{0}[0, T]$, where $F$ is given by equation (4.1). These facts tell us that our assumptions in Section 3 are satisfied. Hence we can apply our results from the previous section to obtain a solution of the diffusion equation for harmonic oscillator (5.1) and of the Schrödinger equation for harmonic oscillator (5.2) which are the limits of the Wiener integrals and of the analytic Feynman integrals, respectively, for the Fourier-type functionals.

(1) The solution of the diffusion equation for harmonic oscillator (5.1) is the limit of the Wiener integrals for the Fourier-type functionals. Let $\varphi$ and $\widehat{V_{m}}$ be given by equations $(2.1)$ and (5.6), respectively. Assume that $\varphi$ is a bounded function. Then the limit of the Wiener integrals for the Fourier-type functionals

$$
\lim _{m \rightarrow \infty} \int_{C_{0}[0, T]} \varphi\left(\lambda^{-\frac{1}{2}} x(T)\right) \widehat{V_{m}}\left(\lambda^{-\frac{1}{2}} x\right) d m(x)
$$

is a solution of the diffusion equation for harmonic oscillator (5.1).

(2) The solution of the Schrödinger equation for harmonic oscillator (5.2) is the limit of the analytic Feynman integrals for the Fourier-type functionals. Let $\varphi$ and $\widehat{V_{m}}$ be given by equations (2.1) and (5.6), respectively. Assume that $\varphi$ is a bounded function. Then

$$
\lim _{m \rightarrow \infty} \int_{C_{0}[0, T]}^{\mathrm{anf}_{1}} \varphi(x(T)) \widehat{V_{m}}(x) d m(x)
$$

is a solution of the Schrödinger equation for harmonic oscillator (5.2). 
(3) Also, we obtain an expression for the solution of the Schrödinger equation for harmonic oscillator (5.2) via the $\diamond$-convolution. Let $\varphi$ and $\widehat{V_{m}}$ be given by equations (2.1) and (5.6), respectively. Assume that $\varphi \in \mathcal{S}\left(\mathbb{R}^{\nu}\right)$ for all $\nu=1,2, \ldots$ and $\widehat{\Phi}$ is a Fourier-type functional so that $\widehat{\Phi}(x)=\varphi(x(T))$. Suppose that $\widehat{\Phi}$ is a Fourier-type functional so that $\widehat{\Phi}(x)=\varphi(x(T))$. Then

$$
\lim _{m \rightarrow \infty}(2 \pi)^{-\frac{m}{2}} \int_{C_{0}[0, T]}^{\operatorname{anf}_{1}}\left(\widehat{\Phi \diamond V_{m}}\right)(x) d m(x)
$$

is also a solution of the Schrödinger equation for harmonic oscillator (5.2).

Now we establish solutions of the diffusion equation and the Schrödinger equation for harmonic oscillator having a special initial condition, but are very useful in quantum mechanics.

Recall the diffusion equation

$$
\frac{\partial}{\partial t} \psi(u, t)=\frac{1}{2 \lambda} \Delta \psi(u, t)-a^{2} u^{2} \psi(u, t)
$$

with the initial condition $\psi(u, 0)=\varphi(u)$. For the present application, we choose the following initial condition

$$
\psi(u, 0)=\varphi(u)= \begin{cases}A, & |u| \leq L / 2, \\ 0, & |u|>L / 2,\end{cases}
$$

where $A$ is a real constant. In view of the Schrödinger equation, this condition corresponds to a pulse wave packet with constant amplitude $A$ in the given range of $|u| \leq L / 2$ [17]. From Lemma 4.1, the solution of the diffusion equation for harmonic oscillator with the given initial condition is

$$
A \lim _{m \rightarrow \infty} \int_{C_{0}[0, T]} \widehat{V_{m}}\left(\lambda^{-\frac{1}{2}} x\right) d m(x) .
$$

Now using equations (2.1) and (5.4), it follows that

$$
\begin{aligned}
\int_{C_{0}[0, T]} \widehat{V_{m}}\left(\lambda^{-\frac{1}{2}} x\right) d m(x) & =\left(\frac{1}{2 \pi}\right)^{\frac{m}{2}} \int_{\mathbb{R}^{m}} \exp \left\{-\frac{a^{2}}{\lambda} \sum_{j=1}^{m} \beta_{j} u_{j}^{2}-\frac{|\vec{u}|^{2}}{2}\right\} d \vec{u} \\
& =\prod_{j=1}^{m}\left(\frac{\lambda}{2 a^{2} \beta_{j}+\lambda}\right) \\
& =\prod_{j=1}^{m}\left(\frac{\left(j-\frac{1}{2}\right)^{2} \pi^{2} \lambda}{2 a^{2} T+\left(j-\frac{1}{2}\right)^{2} \pi^{2} \lambda}\right) .
\end{aligned}
$$

Hence the solution of the diffusion equation for harmonic oscillator with the wave packet reads

$$
A \lim _{m \rightarrow \infty} \prod_{j=1}^{m}\left(\frac{\left(j-\frac{1}{2}\right)^{2} \pi^{2} \lambda}{2 a^{2} T+\left(j-\frac{1}{2}\right)^{2} \pi^{2} \lambda}\right)=A \operatorname{sech}\left(\sqrt{\frac{2 a^{2} T}{\lambda}}\right) .
$$


Furthermore, the solution of the Schrödinger equation for harmonic oscillator with the wave packet is

$$
\begin{aligned}
\lim _{m \rightarrow \infty} \int_{C_{0}[0, T]}^{\operatorname{anf}_{1}} \varphi(x(T)) \widehat{V_{m}}(x) d m(x) & =A \lim _{m \rightarrow \infty} \prod_{j=1}^{m}\left(-\frac{\left(j-\frac{1}{2}\right)^{2} \pi^{2} i}{2 a^{2} T-\left(j-\frac{1}{2}\right)^{2} \pi^{2} i}\right) \\
& =A \operatorname{sech}\left(\sqrt{\frac{2 a^{2} T}{-i}}\right)=A \sec \left(\sqrt{-i 2 a^{2} T}\right) .
\end{aligned}
$$

So far, we have considered the harmonic oscillator. In our next statements, we will introduce another well-known potential in quantum mechanics.

For positive real constants $m, a$, and $\omega$, let

$$
V(u)=\frac{m \omega^{2}}{8 a^{2}}\left(u^{2}-a^{2}\right)^{2} .
$$

This is a double-well potential with two minima at $u= \pm a$ [18]. For sufficiently large $a$, by expanding the potential at $u= \pm a$, one can obtain the following expression:

$$
\begin{aligned}
V(u) & =\frac{m \omega^{2}}{2}(u \mp a)^{2}\left[1 \pm \frac{u \mp a}{a}+\frac{(u \mp a)^{2}}{4 a^{2}}\right] \\
& \simeq \frac{m \omega^{2}}{2}(u \mp a)^{2} .
\end{aligned}
$$

This implies that when $a$ is large, the double-well potential becomes two separate harmonic oscillators at $u= \pm a$, which is discussed in the previous statement. For this special case, we can exploit the results obtained from the previous statement and find solutions of the diffusion equation and the Schrödinger equation for double-well potential with large separation by choosing the same initial condition for $\varphi(u)$.

When $V(u)=\frac{m \omega^{2}}{8 a^{2}}\left(u^{2}-a^{2}\right)^{2}$ in equation (1.1), this equation is called the diffusion equation for double-well potential with two minima at $u= \pm a$

$$
\frac{\partial}{\partial t} \psi(u, t)=\frac{1}{2 \lambda} \Delta \psi(u, t)-\frac{m \omega^{2}}{8 a^{2}}\left(u^{2}-a^{2}\right)^{2} \psi(u, t)
$$

with the initial condition $\psi(u, 0)=\varphi(u)$ and hence the solution of the diffusion equation for double-well potential is given by

$$
\int_{C_{0}[0, T]} \varphi\left(\lambda^{-1 / 2} x(T)\right) \exp \left\{-\frac{m \omega^{2}}{8 a^{2}} \int_{0}^{T}\left(\lambda^{-\frac{1}{2}} x^{2}(s)-a^{2}\right)^{2} d s\right\} d m(x) .
$$

Also, when the time is replaced with the imaginary time, this diffusion equation for the harmonic oscillator (5.1) becomes the Schrödinger equation for the harmonic oscillator

$$
i \frac{\partial}{\partial t} \psi(u, t)=-\frac{1}{2} \Delta \psi(u, t)+\frac{m \omega^{2}}{8 a^{2}}\left(u^{2}-a^{2}\right)^{2} \psi(u, t)
$$

with the initial condition $\psi(u, 0)=\varphi(u)$. 
We note that

$$
x^{4}(t)=\langle\alpha, x\rangle^{4}
$$

where $\alpha(s)=\chi_{[0, t]}(s)$ and so

$$
\int_{0}^{T} x^{4}(s) d s=\int_{0}^{T}\langle\alpha, x\rangle^{4} d s .
$$

Let $\left\{\alpha_{1}, \ldots, \alpha_{n}\right\}$ be as in equation (5.3). Then the set $\left\{\alpha, \alpha_{1}, \ldots, \alpha_{n}\right\}$ may not be an orthonormal set of functions from $L_{2}[0, T]$. But by Gram-Schmidt process, we obtain an orthonormal set $\left\{\alpha_{1}^{\prime}, \ldots, \alpha_{m}^{\prime}\right\}$. In this case, we set $\alpha_{1}^{\prime}=$ $\frac{\alpha}{\|\alpha\|_{2}}$. Also, note that

and so

$$
V(u)=\frac{m \omega^{2}}{8 a^{2}} u^{4}-\frac{m \omega^{2}}{4}+\frac{m \omega^{2} a^{2}}{8}
$$

$$
\begin{aligned}
& \exp \left\{-\int_{0}^{T} V(x(s)) d s\right\} \\
= & \exp \left\{-\frac{m \omega^{2}}{8 a^{2}} \int_{0}^{T} x^{4}(s) d s+\frac{m \omega^{2}}{4} \int_{0}^{T} x^{2}(s) d s+\frac{m \omega^{2} a^{2}}{8}\right\} \\
= & \lim _{m \rightarrow \infty} \exp \left\{-\frac{m \omega^{2}}{8 a^{2}} \int_{0}^{T}\left\langle\alpha_{1}^{\prime}, x\right\rangle^{4} d s+\frac{m \omega^{2}}{4} \sum_{j=2}^{m} \beta_{j}\left\langle\alpha_{j}^{\prime}, x\right\rangle^{2}+\frac{m \omega^{2} a^{2}}{8}\right\} .
\end{aligned}
$$

These facts tell us that our assumptions in Section 3 are satisfied. Hence we can apply our results from the previous section to obtain a solution of the diffusion equation for double-well potential (5.8) and of the Schrödinger equation for double-well potential (5.9) which are the limits of the Wiener integrals and of the analytic Feynman integrals for the Fourier-type functionals as the case of harmonic oscillator.

Finally, we give some additional results as remarks. We will investigate a series representation of the analytic Feynman integral for the Fourier-type functional using hypergeometric series expansion which is different form Taylor series expansion.

The following statement is well-known and plays a key role in Remarks 5.2 and 5.3 below. The hypergeometric function has a hypergeometric series given by

$$
\begin{aligned}
{ }_{1} \mathcal{F}_{1}[a, b, z] \\
=1+\frac{a}{b} z+\frac{a(a+1)}{b(b+1)} \frac{z^{2}}{2 !}+\frac{a(a+1)(a+2)}{b(b+1)(b+2)} \frac{z^{3}}{3 !} \\
+\frac{a(a+1)(a+2)(a+3)}{b(b+1)(b+2)(b+3)} \frac{z^{4}}{4 !}+\cdots=\sum_{s=0}^{\infty} \frac{(a)_{s}}{(b)_{s}} \frac{z^{s}}{s !},
\end{aligned}
$$

where $(a)_{s}$ is a pochhammer symbol, that is,

$$
(a)_{s}=a(a+1)(a+2) \cdots(a+s-1) .
$$


If $a$ and $b$ are integers, $a<0$ and either $b>0$ or $b<a$, then the series yields a polynomial with a finite number of terms. If $b \in \mathbb{Z}^{-} \cup\{0\}$, then ${ }_{1} \mathcal{F}_{1}[a, b, z]$ is undefined.

In Fourier transform theories, it is not easy to evaluate $\widehat{\Delta^{k}} f$ of $f \in \mathcal{S}\left(\mathbb{R}^{n}\right)$. But using equation (2.2), $\widehat{\Delta^{k} f}$ can be evaluated easily whenever $\widehat{f}$ of $f$ is evaluated. Likewise, it also is not easy to evaluate the analytic Feynman integral for $\widehat{\Delta^{k} F}$ of a Fourier-type functional $F$. However, we will use equation (2.6) and the hypergeometric series expansion to easily evaluate the analytic Feynman integral for $\widehat{\Delta^{k} F}$. Equation (5.11) is the series representation of the analytic Feynman integral for the Fourier-type functional $\widehat{\Delta^{k} F}$ using hypergeometric series expansion.

Remark 5.2. Let $\widehat{\Delta^{k} F}$ be given by equation (2.5). Then the analytic Feynman integral for $\widehat{\Delta^{k} F}$ exists and is given by the formula

$$
\begin{aligned}
& \int_{C_{0}[0, T]}^{\operatorname{anf}_{1}} \widehat{\Delta^{k} F}(x) d m(x) \\
& =\left(\frac{\sqrt{-i}}{2 \pi}\right)^{n} \sum_{l_{1}+\cdots+l_{n}=k} \frac{(-1)^{k} k !}{l_{1} ! \cdots l_{n} !} \int_{\mathbb{R}^{n}} f(\vec{u}) \exp \left\{-\frac{i|\vec{u}|^{2}}{2}\right\} \\
& \prod_{j=1}^{n}(2 i)^{l_{j}} \frac{2 \Gamma\left(\frac{1}{2}+l_{j}\right)}{\sqrt{-2 i}}{ }_{1} \mathcal{F}_{1}\left[-l_{j}, \frac{1}{2}, \frac{i u_{j}^{2}}{2}\right] d \vec{u},
\end{aligned}
$$

where ${ }_{1} \mathcal{F}_{1}$ is given by equation $(5.10)$ and $\Gamma$ is the Gamma function.

Equation (5.11) follows from the Multinomial Theorem, the definition of analytic Feynman integral and an integration formula

$$
\int_{\mathbb{R}} \xi^{2 k} \exp \left\{-\frac{\lambda}{2}\left(\xi-\frac{i u}{\lambda}\right)^{2}\right\} d \xi=\left(\frac{2}{\lambda}\right)^{k} \frac{2 \Gamma\left(\frac{1}{2}+k\right)}{\sqrt{2} \sqrt{\lambda}}{ }_{1} \mathcal{F}_{1}\left[-k, \frac{1}{2}, \frac{u^{2}}{2 \lambda}\right] .
$$

We finish this paper by stating an application of equation (5.11).

Remark 5.3. Let $\widehat{V_{m}}$ be given by equation (5.6). Let

$$
i \frac{\partial}{\partial t} \psi(u, t)=-\frac{1}{2} \Delta \psi(u, t)+\Delta^{k} V(u) \psi(u, t)
$$

be a modified Schrödinger equation for a pulse wave packet with constant amplitude introduced in the previous section. Then the solution of the modified Schrödinger equation for harmonic oscillator (5.12) is given by

$$
A \lim _{m \rightarrow \infty} \int_{C_{0}[0, T]}^{\mathrm{anf}_{1}} \widehat{\Delta^{k} V_{m}}(x) d m(x) .
$$


Furthermore, using Remark 5.2, we have

$$
\begin{aligned}
& \int_{C_{0}[0, T]}^{\mathrm{anf}_{1}} \widehat{\widehat{\Delta^{k} V_{m}}}(x) d m(x) \\
= & \left(\frac{\sqrt{-i}}{2 \pi}\right)^{m} \sum_{\substack{l_{1}+\cdots+l_{m}=k \\
m}} \frac{(-1)^{k} k !}{l_{1} ! \cdots l_{m} !} \int_{\mathbb{R}^{m}} V_{m}(\vec{u}) \exp \left\{-\frac{i|\vec{u}|^{2}}{2}\right\} \\
& \prod_{j=1}^{m}(2 i)^{l_{j}} \frac{2 \Gamma\left(\frac{1}{2}+l_{j}\right)}{\sqrt{-2}}{ }_{1} \mathcal{F}_{1}\left[-l_{j}, \frac{1}{2}, \frac{i u_{j}^{2}}{2}\right] d \vec{u} .
\end{aligned}
$$

Equation (5.13) tells us that the solution of the modified Schrödinger equation for harmonic oscillator (5.12) is the limit of the hypergeometric series expansions.

Acknowledgements. The authors would like to express their gratitude to the referees for their valuable comments and suggestions which have improved the original paper.

\section{References}

[1] S. J. Chang, J. G. Choi, and D. Skoug, Evaluation formulas for conditional function space integrals I, Stoch. Anal. Appl. 25 (2007), no. 1, 141-168.

[2] D. M. Chung and S. J. Kang, Evaluation formulas for conditional abstract Wiener integrals, Stoch. Anal. Appl. 7 (1989), no. 2, 125-144.

[3] _ Evaluation formulas for conditional abstract Wiener integrals II, J. Korean Math. Soc. 27 (1990), no. 2, 137-144.

[4] _ Evaluation of some conditional abstract Wiener integrals, Bull. Korean Math. Soc. 26 (1989), no. 2, 151-158.

[5] H. S. Chung and S. J. Chang, Some applications of the spectral theory for the integral transform involving the spectral representation, J. Funct. Space Appl. 2012 (2012), Article ID 573602, 17 pages.

[6] H. S. Chung and V. K. Tuan, Generalized integral transforms and convolution products on function space, Integral Transforms Spec. Funct. 22 (2011), no. 8, 573-586.

[7] _ Fourier-type functionals on Wiener space, Bull. Korean Math. Soc. 49 (2012), no. 3, 609-619.

[8] - A sequential analytic Feynman integral of functionals in $L_{2}\left(C_{0}[0, T]\right)$, Integral Transforms Spec. Funct. 23 (2012), no. 7, 495-502.

[9] R. P. Feynman, Space-time approach to non-relativistic quantum mechanics, Rev. Modern Phys. 20 (1948), 367-387.

[10] G. W. Johnson and M. L. Lapidus, The Feynman Integral and Feynman's Operational Calculus, Clarendon Press, Oxford, 2000.

[11] G. W. Johnson and D. L. Skoug, Scale-invariant measurability in Wiener space, Pacific J. Math. 83 (1979), no. 1, 157-176.

[12] M. Kac, On distributions of certain Wiener functionals, Trans. Amer. Math. Soc. 65 (1949), 1-13.

[13] equations, In: Proc. Second Berkeley Symposium on Mathematical Statistic and Probability(ed. J. Neyman), 189-215, Univ. of California Press, Berkeley, 1951.

[14] _ Probability, Number theory, and Statistical Physics, K. Baclawski and M.D. Donsker (eds.), Mathematicians of Our Time 14 (Cambridge, Mass.-London, 1979). 
[15] - Integration in Function Spaces and Some of its Applications, Lezione Fermiane, Scuola Normale Superiore, Pisa, 1980.

[16] H.-H. Kuo, Gaussian Measure in Banach Space, Lecture Notes in Math. 463, Springer, Berlin, 1975.

[17] E. Merzbacher, Quantum Mechanics 3rd ed., Wiley, NJ (1998), Chap. 5.

[18] C. S. Park, M. G. Jeong, S. K. Yoo, and D. K. Park, Double-well potential: The WKB approximation with phase loss and anharmonicity effect, Phys. Rev. A 58 (1998), 34433447.

[19] B. Simon, Functioanl Integration and Quantum Physis, Academic Press, New York, 1979.

[20] V. K. Tuan, Paley-Wiener type theorems, Frac. Calc. Appl. Anal. 2 (1999), no. 2, 135143.

[21] T. Zastawniak, The equaivalence of two approaches to the Feynman integral for the anharmonic oscillator, Univ. Iagel. Acta Math. 28 (1991), 187-199.

Seung Jun Chang

Department of Mathematics

DANKOOK UNIVERSITY

Cheonan 330-714, Korea

E-mail address: sejchang@dankook.ac.kr

Jae Gil Choi

Department of Mathematics

DANKOOK UNIVERSITY

Cheonan 330-714, Korea

E-mail address: jgchoi@dankook.ac.kr

Hyun Soo Chung

Department of Mathematics

DANKOOK UNIVERSITY

Cheonan 330-714, Korea

E-mail address: hschung@dankook.ac.kr 